\title{
Do Inner Planets Modulate the Space Environment of the Earth?
}

\author{
Jung-Hee Kim, Heon-Young Chang ${ }^{\dagger}$ \\ Department of Astronomy and Atmospheric Sciences, BK21 plus team, Kyungpook National University, Daegu 702-701, Korea
}

Variabilities in the solar wind cause disturbances throughout the heliosphere on all temporal and spatial scales, which leads to changeable space weather. As a view of space weather forecasting, in particular, it is important to know direct and indirect causes modulating the space environment near the Earth in advance. Recently, there are discussions on a role of the interaction of the solar wind with Mercury in affecting the solar wind velocity in the Earth's neighborhood during its inferior conjunctions. In this study we investigate a question of whether other parameters describing the space environment near the Earth are modulated by the inner planets' wake, by examining whether the interplanetary magnetic field and the proton density in the solar wind observed by the Advanced Composition Explorer (ACE) spacecraft, and the geomagnetic field via the Dst index and Auroral Electrojet index (AE index) are dependent upon the relative position of the inner planets. We find there are indeed apparent variations. For example, the mean variations of the geomagnetic fields measured in the Earth's neighborhood apparently have varied with a timescale of about 10 to 25 days. Those variations in the parameters we have studied, however, turn out to be a part of random fluctuations and have nothing to do with the relative position of inner planets. Moreover, it is found that variations of the proton density in the solar wind, the Dst index, and the AE index are distributed with the Gaussian distribution. Finally, we point out that some of properties in the behavior of the random fluctuation are to be studied.

Keywords: solar wind, Mercury, Venus, space environment

\section{INTRODUCTION}

The solar wind originating from the surface of the Sun, as turbulent plasma, carries the magnetic field lines. The solar wind with the magnetic field rules the heliosphere and interacts with solar system bodies in the course of propagation. For instance, geomagnetic disturbances occur by the Dungey mechanism when an interplanetary coronal mass ejection (ICME) blob or magnetic clouds engulfs the Earth, provided the ICME blob or the cloud has a magnetic field B with a significant negative Bz component (Dungey 1961). Reconnection occurs at the day-side magnetopause between the terrestrial magnetic field and the interplanetary field. When the reconnected field lines are swept back into the geomagnetic tail in the night side, a neutral point may form through which charged particles in the solar wind enter into the terrestrial magnetosphere. Low-energy particles spiral around the open field lines and impinge on the terrestrial atmosphere in the polar regions, causing enhanced aurora. High-energy particles are deflected around the Earth in circular orbits in the equatorial plane, forming the ring current at several Earth radii. It causes large geomagnetic field reductions at the ground, which are measured by the disturbance storm time index (Dst; Sugiura 1964). To quantify the solar wind-magnetosphere interaction, the geoeffectiveness and geomagnetic impacts of the solar wind's interplanetary structures had been widely studied in the last decades, with in situ data from several spacecraft observations and ground-based data from geomagnetic and upper atmosphere stations spread worldwide (Akasofu 1981, Gonzalez et al. 1994, Cane 2000, Echer et al. 2005, Xu et al. 2009, Kane 2010, Cerrato et al. 2012, Olyak 2012, Oh \& Chang 2012). In addition, Galactic cosmic rays (GCRs) entering the heliosphere are modulated by the solar wind plasma and the associated magnetic field (Dumbović, et al. 2011, 2012, Singh et al. 2012, Cao et al. (c) This is an open Access article distributed under the terms of the Creative Commons Attribution Non-Commercial License (http:// creativecommons.org/licenses/by-nc/3.0/) which permits unrestricted non-commercial use, distribution, and reproduction in any medium, provided the original work is properly cited.
Received Feb 3, 2014 Revised Feb 20, 2014 Accepted Feb 22, 2014 †Corresponding Author

E-mail: hyc@knu.ac.kr

Tel: +82-53-950-6367, Fax: +82-53-950-6359 
2013).

Recently, Nikulin (2013) studied the interaction of the solar wind with Mercury using the mean daily velocity of the solar wind of 54 cases in the period from 1995 to 2012 observed advanced composition explorer (ACE) data by the superimposed epoch method. This method of analysis is originally proposed by Chree (1913). Nikulin (2013) found a noticeable increase both before and after the inferior conjunctions with Mercury as well as decrease in the velocity of the solar wind around the inferior conjunctions. He consequently associated his finding with Mercury's wake, or the shadow. He also suggested that the general tendencies are less expressed at the stage of the ascending phase of the solar cycle 23 than those of the descending phase of the solar cycle 23 and the ascending phase of the solar cycle 24 . More recently, however, Kim \& Chang (2014) have disputed his conclusions by analyzing the daily velocity of the solar wind for periods of the superior conjunctions with Mercury and randomly chosen time series regardless of Mercury's relative position with respect to the Earth. They have also demonstrated that their findings can be reproduced with the cases of Venus. They have concluded that the relative position of the inner planets are nothing special in the sense that these characteristic features are omnipresent and that noticed modulation of the solar wind velocity is a part of random fluctuations of the solar wind velocity in the course of its propagation in the interplanetary space rather than one associated with the interaction with a flow of the solar wind with the inner planets. In this study we investigate a question of whether other parameters describing the space environment near the Earth are modulated by the inner planets' wake. This question is of prime importance since it has implications both on the plasma physics of the interplanetary space and on the space weather forecast. Even though Kim \& Chang (2014) have shown that the variation in the solar wind velocity turns out to be a part of random fluctuations, this does not necessarily mean an impossibility of survival of the hermean trace to the distance of 1 AU from the Sun in the Earth's neighborhood during inferior conjunctions with the inner planets. That is, one should only be able to consider that the solar wind velocity is not a proper observable modulated by the inner planets at the distance of $1 \mathrm{AU}$ from the Sun. We are motivated by this line of argument and study by examining whether some of properties of the interplanetary magnetic field and the proton density in the solar wind observed by the ACE spacecraft, and of the geomagnetic field are subject to the relative position of the inner planets. This paper is organized as follows. We begin with brief descriptions of data analyzed for the present paper in Section 2. We present and discuss results based on the test with various parameters in diverse cases in Section 3. Finally, we summarize and conclude in Section 4.

\section{DATA}

For the present analysis, firstly, we have used the interplanetary magnetic field data observed during the period from 1997 to 2013 by the Magnetic Field Experiment (MAG) on the ACE spacecraft, which consists of twin vector fluxgate magnetometers controlled by a common CPU. The sensors are mounted on booms extending 4.19 meters from the center of the spacecraft at opposite sides along the $\pm \mathrm{Y}$ axes of the spacecraft. The instrument returns 6 magnetic field vector measurements each second, divided between the two sensors, with onboard snapshot and FFT buffers to enhance the high-frequency resolution. Magnetic field vectors are given in the Radial Tangential Normal (RTN), Geocentric Solar Ecliptic (GSE), and Geocentric Solar Magnetospheric (GSM) coordinate systems. For this particular analysis, we adopt field values in the GSM coordinate system, where the $\mathrm{X}$-axis is parallel to the EarthSun line and $\mathrm{Z}$-axis is the projection of dipole axis on the YZ plane of the GSE coordinate system, the Z-axis of the GSE coordinate system being the ecliptic north pole. Data have been taken from the ACE Science Center website ${ }^{1}$. Secondly, we have used the number density of protons ( $\mathrm{np}$ in $\# \mathrm{~cm}^{-3}$ ) in the solar wind during the period from 1998 to 2013. The proton density is observed by the Solar Wind Electron, Proton, and Alpha Monitor (SWEPAM) on the ACE spacecraft. We have taken data from the ACE Science Center website. Electron and ion measurements are made with separate sensors of the SWEPAM. The ion sensor measures particle energies between about 0.26 and $36 \mathrm{keV}$, and the electron sensor's energy range is between 1 and $1350 \mathrm{eV}$. Both sensors use electrostatic analyzers with fan-shaped fields-of-view. The electrostatic analyzers measure the energy per charge of each particle by bending their flight paths through the system. The fields-of-view are swept across all solar wind directions by the rotation of the spacecraft. Finally, we have used the Dst index and the Auroral Electrojet index (AE index) observed during the period from 1998 to 2013, in examining properties of the geomagnetic field. The Dst index is one of indices showing the geomagnetic activity, which is derived from a network of near-equatorial geomagnetic observatories that measures the intensity of the ring current. The Dst index from 1957

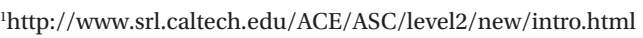


to the present is maintained at National Geophysical Data Center (NGDC) ${ }^{2}$ and is available via $\mathrm{FTP}^{3}$. The AE index is, on the other hand, designed to provide a global, quantitative measure of auroral zone magnetic activity produced by enhanced ionospheric currents flowing below and within the auroral oval. Ideally, it is the total range of deviation at an instant of time from quiet day values of the horizontal magnetic field around the auroral oval. The AE index has been usefully employed both qualitatively and quantitatively as a correlative index in studies of substorm morphology, the behavior of communication satellites, radio propagation, radio scintillation, and the coupling between the interplanetary magnetic field and the Earth's magnetosphere. The AE index is also available via $\mathrm{FTP}^{4}$ of NGDC sites.

\section{RESULTS AND DISCUSSION}

In Fig. 1, we show variations in magnitudes of the interplanetary magnetic field and of its three components of the GSM coordinate system in nT, which are measured in L1 Lagrangian point in the vicinity of the Earth by the ACE spacecraft. From top to bottom, the variations of the mean magnitude of the interplanetary magnetic field, the X-, Y-, Z-components of the magnetic field vector in the GSM coordinate system are presented, respectively. To examine the effect suggested by Nikulin (2013), we have extracted the time series of data for periods of inferior conjunctions with Mercury from 1997 to 2013, whose length of each time series is 29 days. We have marked the epoch of the inferior conjunction with Mercury from the website of Korea Astronomy and Space Science Institute (KASI) ${ }^{5}$. By doing so, we end up with 50 data strings whose length is 29 days. Each of the data string is centered (i.e., $\mathrm{T}=0$ ) at the day when Mercury locates at the inferior conjunction. We superimpose 50 data strings and then average them so that the variation of the mean magnetic field strength is obtained during the inferior conjunctions with Mercury. This is the superimposed epoch method originally suggested by Chree (1913). Apparently, some ups and downs around the epoch of the inferior conjunction can be seen in Fig. 1. Interestingly enough, the time scale of max-to-max or minto-min that can be found in each panel seems quite similar to that seen in Kim \& Chang (2014), which is about 10 to 15

${ }^{2}$ http://ngdc.noaa.gov/

${ }^{3} \mathrm{ftp}$ ://ftp.ngdc.noaa.gov/STP/GEOMAGNETIC_DATA/INDICES/DST ${ }^{4} \mathrm{ftp} / / / \mathrm{ftp}$. ngdc.noaa.gov/STP/GEOMAGNETIC_DATA/AURORAL_ELECTROJET/ ${ }^{5}$ http://www.kasi.re.kr

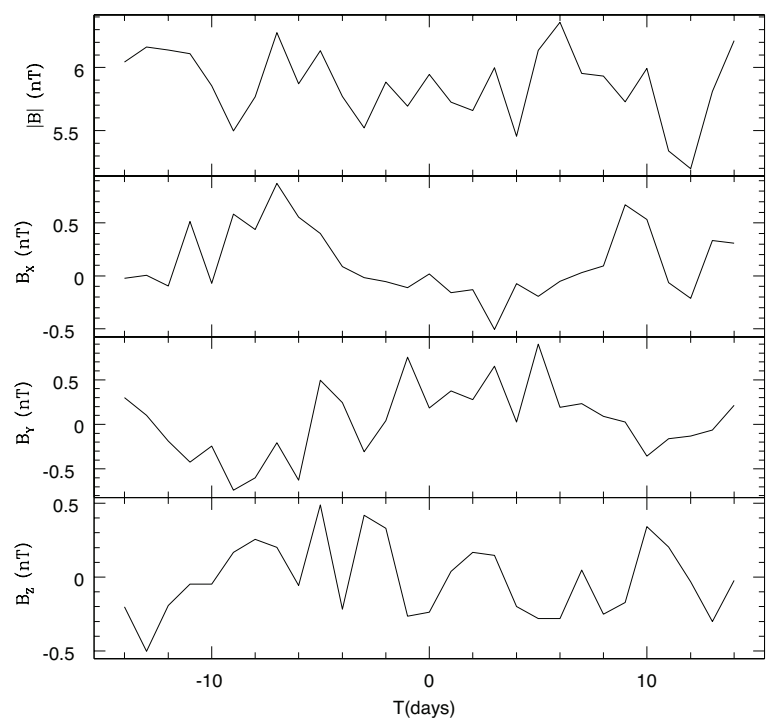

Fig. 1. Variations in magnitudes of the interplanetary magnetic field and of its three components of the GSM coordinate system in nT measured in L1 Lagrangian point in the vicinity of the Earth by the ACE spacecraft. From top to bottom, the mean magnitude of the interplanetary magnetic field, the $X$-, $\mathrm{Y}$-, Z-components of the magnetic field vector in the GSM coordinate system, respectively. Each data string is centered (i.e., $T=0$ ) at the day when Mercury locates at the inferior conjunction and superimposed for averaging over 50 events.

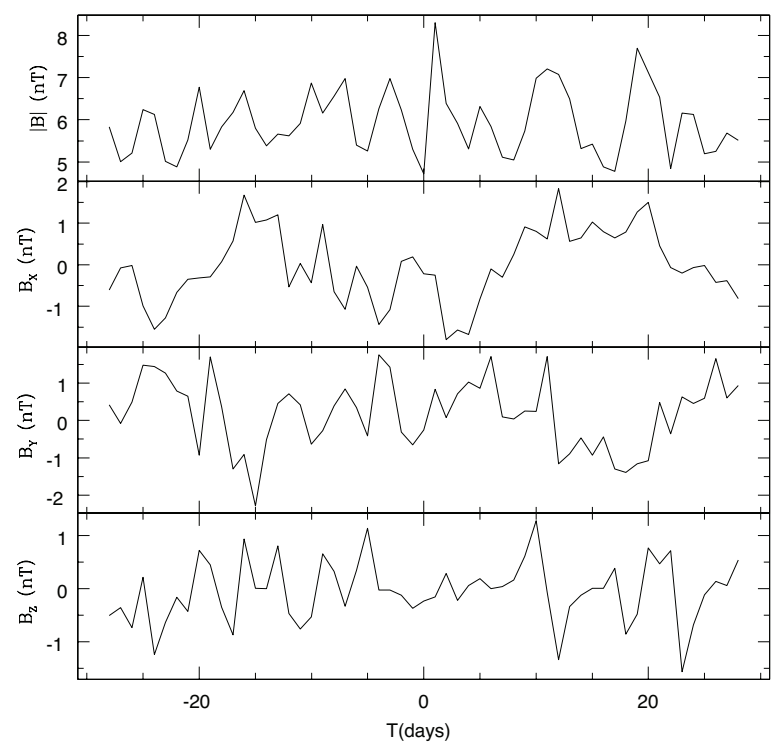

Fig. 2. Similar to Fig. 1, except that results are from data strings for the periods of the inferior conjunctions with Venus. We have 9 cases of the inferior conjunctions with Venus.

days.

In Fig. 2, for comparison, we show similar plots, which result from data for 9 cases of inferior conjunctions with Venus. The time series of data is with a length of 57 days. We have again marked the epoch of the inferior/superior conjunction with Venus from the website of KASI. The ups 


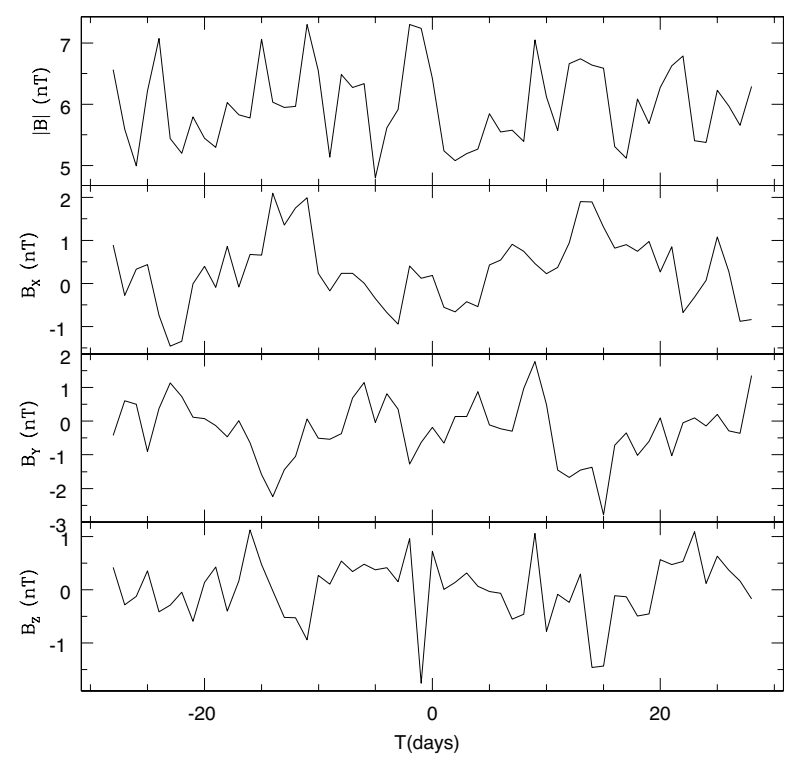

Fig. 3. Similar to Fig. 1, except that results are from data strings for the periods of the superior conjunctions with Venus. We have 10 cases of the superior conjunctions with Venus.

and downs with a period of $\sim 25$ days can be clearly seen in the second and the third panels, though less clear in the first and the last panels. A similar appearance suggests that it has nothing to do with the relative position of the inner planets with respect to the Earth. To compare with cases of inferior conjunctions, in Fig. 3, we show results from data for periods of the superior conjunctions with Venus, where we have 10 cases of the superior conjunctions with Venus. Basically, same main features can be seen in Fig. 3 as shown in Fig. 2. Magnetic field strengths measured in the vicinity of the Earth are also considered as a random fluctuation as in the solar wind velocity. In Fig. 4, we show the mean variations over 9 randomly chosen data strings regardless of Venus' relative position with respect to the Earth, which turn out to be very similar to plots in Figs. 1 to 3 . On the basis of what we have observed in Figs. 1 to 4, we come to a conclusions that the effect suggested by Nikulin(2013), in which a trace of an inner planet sweeps the near-Earth space and somehow disturbs its environment, cannot be found in the planetary magnetic field strength. Instead all one can see seems a characteristic feature of random fluctuations.

In Fig. 5, we show variations in the number density of protons in the solar wind measured by the ACE spacecraft. In the top panel, we show results from data strings for 9 cases of the inferior conjunctions with Venus. In the middle and the bottom panels, results are from data strings for 10 cases of superior conjunctions with Venus and for 9 cases of randomly chosen occurrences, respectively. Instead of showing characteristic features seen in Figs. 1 to 4 ,

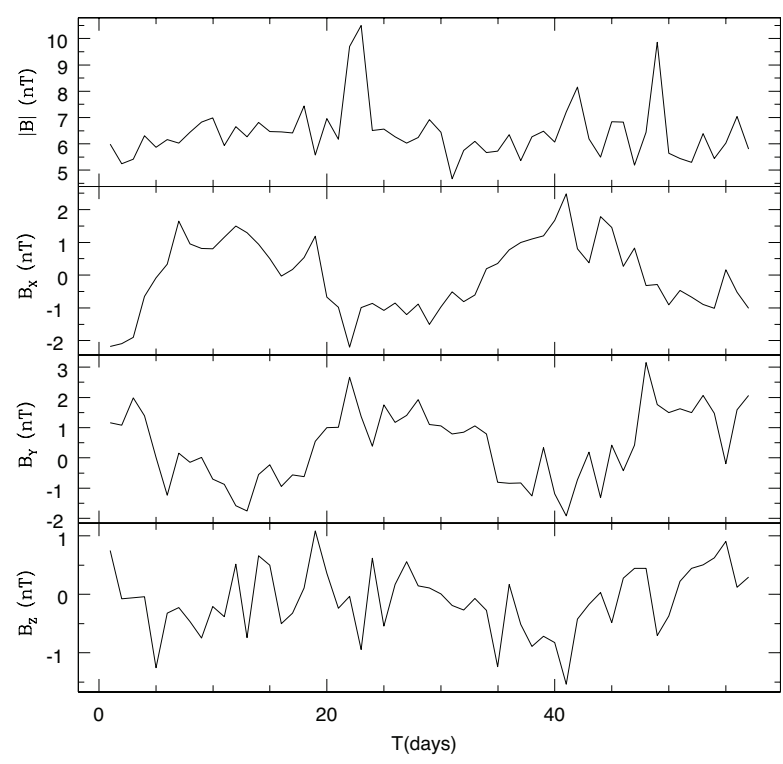

Fig. 4. Similar to Fig. 1, except resulting from the magnetic fields over 9 randomly chosen data strings regardless of Venus' relative position with respect to the Earth.

variations in Fig. 5 look like a randomly distributed noise with respect to a mean value, though it is hard to definitely tell since the length of time series of data is insufficiently long. In order to test its randomness we count the number of the proton density values in the solar wind in each density bin. We subsequently construct a histogram due to the proton number density. We then fit the Gaussian function to the histogram by independently adjusting 3 parameters assuming a normal distribution of errors. In Fig. 6, we show corresponding histograms of the proton density and their best fit obtained using the Gaussian function. From top to bottom, the histograms are obtained from the variation in the number density of the case of the inferior conjunctions with Venus, the case of the superior conjunctions, the randomly chosen case, respectively. Thick curves represent the best fit of the Gaussian function. As shown in Fig. 6 , the distribution of the proton density as a whole is the Gaussian distribution. Furthermore, resulting three Gaussian functions look similar regardless of how they are constructed. As a result, again, we conclude that the relative position of the inner planets does not affect the mean variation in the proton's number density in the solar wind observed in the Earth's neighborhood. We carry on the same analysis with the Dst index and the AE index, in turn. In Fig. 7, we show variations in the Dst index, which are obtained by the superimposed epoch method, from three different criteria. From top to bottom, we show results from cases of the inferior conjunctions and superior conjunctions with Venus, and randomly chosen occurrences, respectively. In 


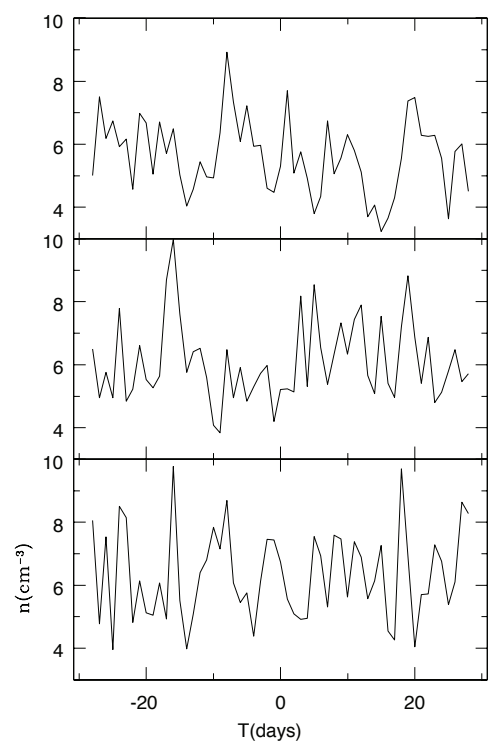

Fig. 5. Variations in the number density of the solar wind protons measured by the ACE spacecraft. In the top panel, we show results from data strings for 9 cases of inferior conjunctions with Venus. In the middle and the bottom panels, results are from data strings for 10 cases of superior conjunctions with Venus and for 9 cases of randomly chosen occurrences.

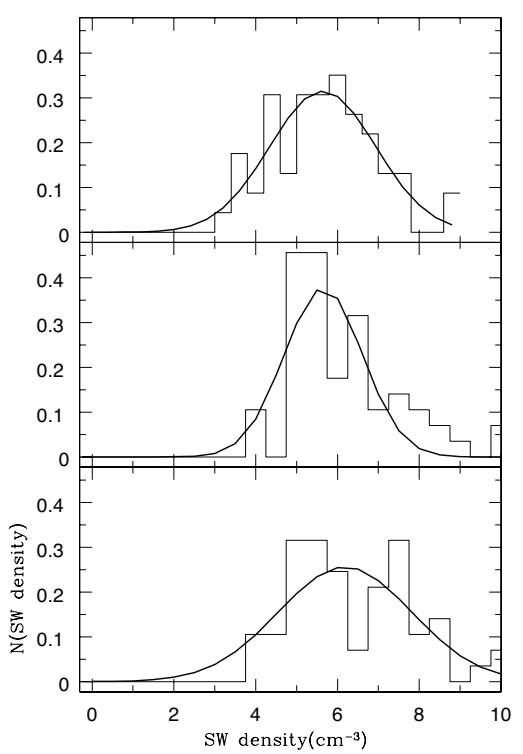

Fig. 6. Histograms of the number density of protons in the solar wind and their best fits obtained using the Gaussian function. From top to bottom, the histograms are obtained from the variation in the number density of the case of the inferior conjunctions with Venus, the case of the superior conjunctions, the randomly chosen case, respectively. Thick curves represent the best fit of the Gaussian function.

Fig. 8, we too show the histograms of the Dst index and their best fit obtained using the Gaussian function. From top to bottom, the histograms are obtained from the variation in the Dst index of the case of the inferior conjunctions with Venus, the case of the superior conjunctions, the randomly

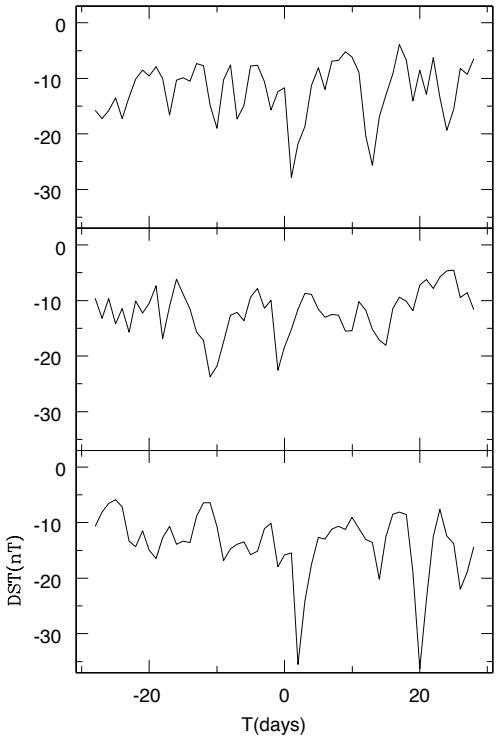

Fig. 7. Similar to Fig. 5, except that we show variations in the Dst index.

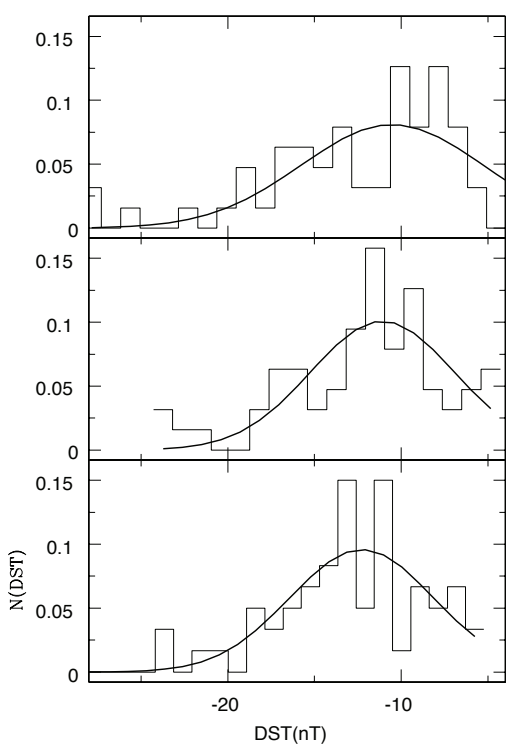

Fig. 8. Similar to Fig. 6, except that we show the histograms of the Dst index and their best fits.

chosen case, respectively. Thick curves represent the best fit of the Gaussian function. We conclude the same. That is, the relative position of the inner planets has nothing to do with the variation in the Dst index. In fact, this is what one may obviously expect from the fact that Bz plots show nothing special in plots like Figs. 1 to 4 to show dumb negative $\mathrm{Bz}$ components, reminding the Dst index is sensitively responding to the negative $\mathrm{Bz}$ component. Similarly, in Fig. 9, we show variations in the AE index obtained with three different criteria. In Fig. 10, we show corresponding 


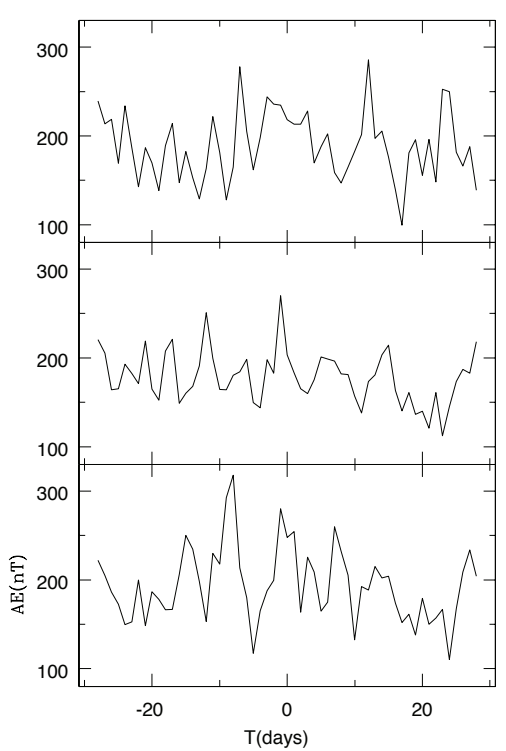

Fig. 9. Similar to Fig. 5, except that we show variations in the AE index.

histograms of the AE index and their best fit obtained using the Gaussian function. From top to bottom, the histograms are obtained from the variation in the AE index of the case of the inferior conjunctions with Venus, the case of the superior conjunctions, the randomly chosen case, respectively. Based on the apparent similarity of the best fits shown in thick curves, we come to the same conclusion as we have done in other parameters. In other words, the relative position of the inner planets does not modulate the $\mathrm{AE}$ index, either.

\section{SUMMARY AND CONCLUSION}

Variabilities in the solar wind in terms of velocity, proton density, interplanetary magnetic fields, lead to interesting phenomena throughout the heliosphere on all temporal and spatial scales. As a view of space weather forecasting, many works are part of a major effort to predict geomagnetic storms. To be able to do so, it is important to know direct and indirect causes to modulate the space environment near the Earth beforehand. Recently, it has been suggested that the interaction of the solar wind with Mercury may affect the solar wind velocity in the Earth's neighborhood during its inferior conjunctions. In this study we investigate a question of whether other parameters describing the space environment near the Earth are modulated by the inner planets' wake, by examining whether properties of the interplanetary magnetic field and the proton density in the solar wind observed by the ACE spacecraft, and of the

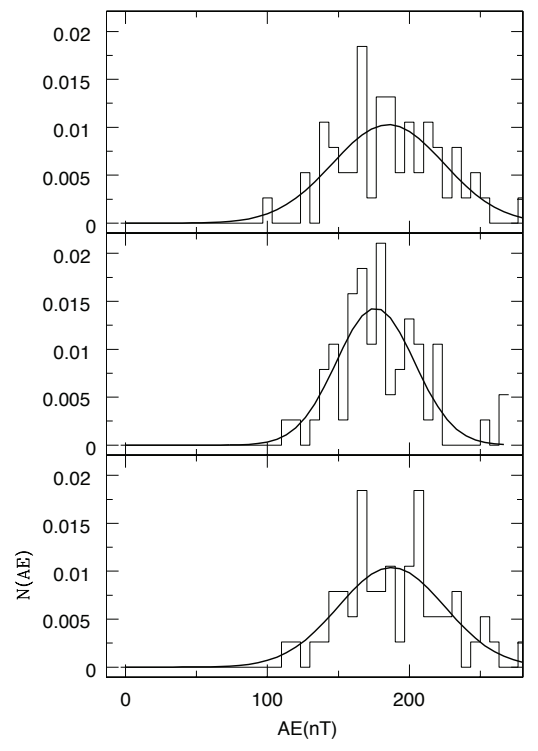

Fig. 10. Similar to Fig. 6, except that we show the histograms of the $A E$ index and their best fits.

geomagnetic field are subject to the relative position of the inner planets.

Our results confirm that those variations in the parameters we have studied here turn out to be a part of random fluctuations and have nothing to do with the relative position of the inner planets. It has been found that minima and maxima in the mean variations of the geomagnetic fields measured in the Earth's neighborhood repeatedly occur with a timescale of about 10 to 25 days. Variations of the proton density in the solar wind, the Dst index, and the $\mathrm{AE}$ index are distributed with the Gaussian distribution. One might wish to perform a systematic statistical study of the characteristics of the distribution. We conclude, finally, that the inner planets cannot affect the Earth's neighborhood during its inferior conjunctions and the observed variations are randomly distributed noise, whose behavior can be further studied in terms of the distribution and/or in the frequency domain.

\section{ACKNOWLEDGMENTS}

We thank the anonymous referees for critical comments and helpful suggestions which greatly improve the original version of the manuscript. This work was supported by BK21 Plus of National Research Foundation of Korea. HYC was supported by the National Research Foundation of Korea Grant funded by the Korean government (NRF2013K2A2A2000525). 


\section{REFERENCES}

Akasofu SI, Energy Coupling between the Solar Wind and the Magnetosphere, Space Sci. Rev., 28, 121-190 (1981). http://dx.doi.org/10.1007/BF00218810

Cane HV, Coronal Mass Ejections and Forbush Decreases, Space Sci. Rev., 93, 55-77 (2000). http://dx.doi.org/ 10.1023/A:1026532125747

Cao J, Duan A, Reme H, Dandouras I, Relations of the Energetic Proton Fluxes in the Central Plasma Sheet with Solar Wind and Geomagnetic Activities, JGR, 118, 7226-7236 (2013). http://dx.doi.org/ 10.1002/2013JA019289

Cerrato Y, Saiz E, Cid C, Gonzalez WD, Palacios J, Solar and Interplanetary Triggers of the Largest Dst Variations of the Solar Cycle 23, JASTP, 80, 111-123 (2012). http:// dx.doi.org/10.1016/j.jastp.2011.09.001

Chree C, Some Phenomena of Sunspots and of Terrestrial Magnetism at Kew Observatory, Philosophical Transactions Royal Society London Series A, 212, 75116 (1913). http://dx.doi.org/10.1098/rsta.1913.0003

Dumbović M, Vršnak B, Čalogović J, Karkica M, Cosmic Ray Modulation by Solar Wind Disturbances, A\&A, 531, A91 (2011). http://dx.doi.org/10.1051/0004$6361 / 201016006$

Dumbović M, Vršnak B, Čalogović J, Župan R, Cosmic Ray Modulation by Different Types of Solar Wind Disturbances, A\&A, 538, A28 (2012). http://dx.doi. org/10.1051/0004-6361/201117710

Dungey JW, Interplanetary Magnetic Field and the Auroral Zones, Phys. Rev. Letters, 6, 47-48 (1961). http://dx.doi. org/10.1103/PhysRevLett.6.47

Echer E, Gonzalez WD, Guarnieri FL, DalLago A, Vieira LEA, Introduction to Space Weather, Adv. Space Res., 35, 855865 (2005). http://dx.doi.org/10.1016/j.asr.2005.02.098

Gonzalez WD, Joselyn JA, Kamide Y, Kroehl HW, Rostoker G, et al., What is a Geomagnetic Storm?, JGR, 99, 57715792 (1994). http://dx.doi.org/10.1029/93JA02867

Kane RP, Relationship between the Geomagnetic Dst (min) and the Interplanetary Bz (min) during Cycle 23, Planet. Space Sci., 58, 392-400 (2010). http://dx.doi. org/10.1016/j.pss.2009.11.005

Kim JH, Chang HY, Do Inner Planets Modulate the Solar Wind Velocity at 1 AU from the Sun?, JASS, 31, 1-6 (2014). http://dx.doi.org/ 10.5140/JASS.2014.31.1.1

Nikulin, IF, Modulation of the Solar Wind Velocity by Mercury, submitted to Planet. Space Sci. (2013).

Oh SJ, Chang HY, Change of Sunspot Groups Observed from 2002 to 2011 at Butter Star Observatory, JASS, 29, 245-251 (2012). http://dx.doi.org/10.5140/JASS.2012.29.3.245
Olyak MR, Large-scale Structure of Solar Wind and Geomagnetic Phenomena, JASTP, 86, 34-40 (2012). http://dx.doi.org/10.1016/j.jastp.2012.06.011

Singh YP, Gautam S, Badruddin, Temporal Variations of Short-and Mid-term Periodicities in Solar Wind Parameters and Cosmic Ray Intensity, JASTP, 89, 48-53 (2012). http://dx.doi.org/10.1016/j.jastp.2012.07.011

Sugiura M, Hourly Values of the Equatorial Dst for IGY, Annales of the International Geophysical Year 35 (Pergamon Press, Oxford, 1964), 945-948.

XuD, Chen T, Zhang XX, Liu Z, Statistical Relationship between Solar Wind Conditions and Geomagnetic Storms in 1998-2008, Planet. Space Sci., 57, 1500-1513 (2009). http://dx.doi.org/10.1016/j.pss.2009.07.015 